Pacific Journal of Mathematics

CONCERNING BOUNDARY VALUE PROBLEMS 


\section{CONCERNING BOUNDARY VALUE PROBLEMS 1}

\section{J. W. NEUBERGER}

1. Introduction. This paper follows work on integral equations by H. S. Wall [4], [5], J. S. MacNerney [1], [2] and the present author [3]. Some results of these papers are used here to investigate certain boundary value problems.

In $\S 2$, results of Wall and MacNerney are used to study a linear boundary value problem which includes problems of the following kind: Suppose that each of $a_{i j}, i, j=1, \cdots, n$ is a continuous function, $a$ and $b$ are numbers and each of $b_{i j}, c_{i j}$ and $d_{i}, i, j=1, \cdots, n$ is a number. Is there a unique function $n$-tuple $f_{1}, \cdots, f_{n}$ such that

$$
f_{i}^{\prime}=\sum_{j=1}^{n} a_{i j} f_{j} \text { and } \sum_{j=1}^{n}\left[b_{i j} f_{j}(a)+c_{i j} f_{j}(b)\right]=d_{i}, \quad i=1, \cdots, n ?
$$

Section 3 contains some observations concerning a nonlinear boundary value problem which includes the problem of solving a certain system of nonlinear first order differential equations together with a nonlinear boundary condition. An example is given in the final section.

$S$ denotes a normed, complete, abelian group (norms are denoted by $\|\cdot\|)$. $B$ denotes the normed, complete, abelian group of all bounded endomorphisms from $S$ to $S$ (the norm of an element $T$ of $B$ is the g.l.b. of the set of all $M$ such that $\|T x\| \leqq M\|x\|$ for all $x$ in $S$ ). $B^{*}$ denotes the set to which $T$ belongs only if $T$ is a continuous function from $S$ to $S$. If $[a, b]$ denotes a number interval, then $C_{[a, b]}$ denotes the set to which $f$ belongs only if $f$ is a continuous function from $[a, b]$ to $S$. The identity function on the numbers is denoted by $j$.

The reader is referred to [1] for a definition of the integral of a function from a number interval $[a, b]$ to $B$ with respect to a function from $[a, b]$ to $B$ and to [3] for a definition of the integral of a function from $[a, b]$ to $S$ with respect to a function from $[a, b]$ to $B^{*}$. [1] and [3] contain existence theorems for these integrals and a discussion of some of their properties.

2. A linear boundary value problem. Suppose that $[a, b]$ is a number interval and $F$ is a continuous function from $[a, b]$ to $B$ which is of bounded variation on $[a, b]$. The following are theorems:

(i) There is a unique continuous function $M$ from $[a, b] \times[a, b]$ to $B$ such that $M(t, u)=I+\int_{u}^{t} d F \cdot M(j, u)$ for each of $t$ and $u$ in $[a, b]$. ( $I$ denotes the identity element in $B$ )

Received March 25, 1959, and in revised form January 22, 1960.

1 Presented to the Society in part, August, 1958, and in part, January, 1959. 
(ii) $M(t, u) M(u, v)=M(t, v)$ if each of $t, u$ and $v$ is in $[a, b]$.

(iii) If $h$ is a continuous function from $[a, b]$ to $S$ and $c$ is in $[a, b]$, then the only element $X$ of $C_{[a, b]}$ such that $X(t)=h(t)+\int_{c}^{t} d F \cdot X$ for each $t$ in $[a, b]$ is given by $X(t)=M(t, c) h(c)+\int_{c}^{t} M(t, j) d h$ for each $t$ in $[a, b] .^{2}$

THEOREM A. Suppose that $H$ is a function from $[a, b]$ to $B$ which is of bounded variation on $[a, b]$. A necessary and sufficient condition that there be a unique element $Y$ of $C_{[a, b]}$ such that

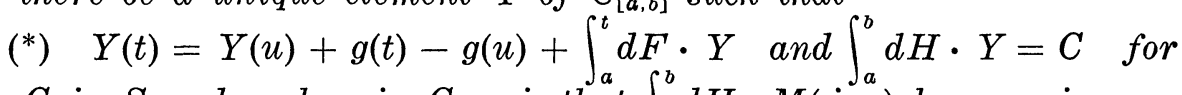
each $C$ in $S$ and each $g$ in $C_{[a, b]}$ is that $\int_{a}^{b} d H \cdot M(j, a)$ have an inverse which is from $S$ onto $S$.

Proof. Consider first the following lemma. If $Y$ is in $C_{[a, b]}$ and satisfies $\left(^{*}\right)$ for each of $u$ and $t$ in $[a, b]$, then

$$
\left[\int_{a}^{b} d H \cdot M(j, a)\right] Y(a)=C-\int_{a}^{b}\left[\int_{j}^{b} d H(s) \cdot M(s, j)\right] d g .
$$

Suppose $Y$ is in $C_{[a, b]}$ and satisfies (*) for each of $u$ and $t$ in $[a, b]$. By (iii), $Y(t)=M(t, a) Y(a)+\int_{a}^{t} M(t, j) d g$ for each $t$ in $[a, b]$ and thus

$$
\begin{aligned}
C & =\int_{a}^{b} d H \cdot Y=\left[\int_{a}^{b} d H \cdot M(j, a)\right] Y(a)+\int_{a}^{b} d H(s) \cdot\left[\int_{a}^{s} M(s, j) d g\right] \\
& =\left[\int_{a}^{b} d H \cdot M(j, a)\right] Y(a)+\int_{a}^{b}\left[\int_{j}^{b} d H(s) \cdot M(s, j)\right] d g \cdot^{3}
\end{aligned}
$$

Hence,

$$
\left[\int_{a}^{b} d H \cdot M(j, a)\right] Y(a)=C-\int_{a}^{b}\left[\int_{j}^{b} d H(s) \cdot M(s, j)\right] d g .
$$

Denote $\int_{a}^{b} d H \cdot M(j, a)$ by $Q$. Suppose that $\left(^{*}\right)$ has a unique solution for each $g$ in $C_{[a, b]}$ and each $C$ in $S$.

Denote by $W$ a point of $S$, by $g$ an element of $C_{[a, b]}$,

2 Certain essential ideas for Theorems (i) and (ii) were given by Wall in [4]. In [5,] Wall gave these theorems for $S$ an $n$-dimensional Euclidean space or suitable infinite dimensional space. In [1], MacNerney extended Wall's theory in proving these theorems for any normed, linear and complete space. Modifications of MacNerney's proofs to the case of $S$ a normed, complete, abelian group are so slight that the proofs are omitted. Discussion concerning the properties and computation of $M$ can be found in each paper listed as reference to this paper.

${ }^{3}$ A proof that $\int_{a}^{b} d H(s) \cdot\left[\int_{a}^{s} M(s, j) d g\right]=\int_{a}^{b}\left[\int_{j}^{b} d H(s) \cdot M(s, j)\right] d g$ which follows closely a similar argument for ordinary integrals, is ommitted. 


$$
W+\int_{a}^{b}\left[\int_{j}^{b} d H(s) \cdot M(s, j)\right] d g
$$

by $C$ and by $X$ the unique element of $C_{[a, b]}$ satisfying $\left(^{*}\right)$ for this $g$ and $C$. By the above lemma, $Q X(a)=C-\int_{a}^{b}\left[\int_{j}^{b} d H(s) \cdot M(s, j)\right] d g=W$. Thus each point of $S$ is the image of some point of $S$ under $Q$, that is, $Q$ takes $S$ onto $S$.

Suppose that $Q$ is not reversible and denote by each of $W, U$ and $V$ a point in $S$ such that $Q U=W, Q V=W$ and $U \neq V$. Denote by $Y$ and $Z$ two elements of $C_{[a, b]}$ such that $Y(t)=U+g(t)-g(a)+$ $\int_{a}^{t} d F \cdot Y$ and $Z(t)=V+g(t)-g(a)+\int_{a}^{t} d F \cdot Z$ for each $t$ in $[a, b]$. Thus, $Y(t)=Y(u)+g(t)-g(u)+\int_{u}^{t} d F^{a} \cdot Y$ and $Z(t)=Z(u)+g(t)-$ $g(u)+\int_{u}^{t} d F \cdot Z$, for each of $u$ and $t$ in $[a, b]$. Since $Y(a)=U$ and $Z(a)=\stackrel{v}{V}$, it follows that $Y \neq Z$. As in the proof of the lemma,

$$
\int_{a}^{b} d H \cdot Y=Q U+\int_{a}^{b}\left[\int_{j}^{b} d H(s) \cdot M(s, j)\right] d g
$$

and

$$
\int_{a}^{b} d H \cdot Z=Q V+\int_{a}^{b}\left[\int_{j}^{b} d H(s) \cdot M(s, j)\right] d g
$$

and so

$$
\int_{a}^{b} d H \cdot Y=\int_{a}^{b} d H \cdot Z,
$$

which means that there is a boundary value problem of the type $\left(^{*}\right)$ which has two solutions, which contradicts the above assumption. Thus if $\left(^{*}\right)$ has a unique solution for each $g$ in $C_{[a, b]}$ and each $C$ in $S, Q$ takes $S$ onto $S$ reversibly.

Suppose that $Q$ takes $S$ onto $S$ reversibly. Denote by $g$ an element of $C_{[a, b]}$ and by $C$ a point in $S$. Denote

$$
\left[\int_{a}^{b} d H \cdot M(j, a)\right]^{-1}\left\{C-\int_{a}^{b}\left[\int_{j}^{b} d H(s) \cdot M(s, j)\right] d g\right\}
$$

by $U$ and denote by $X$ the element of $C_{[a, b]}$ such that $X(t)=U+g(t)-$ $g(a)+\int_{a}^{t} d H \cdot X$ for each $t$ in $[a, b]$. Noting that $X(t)=X(u)+$ $g(t)-g(u)+\int_{u}^{t} d H \cdot X$ and that $X(t)=M(t, a) U+\int_{a}^{t} M(t, j) d g$ for each of $u$ and $t$ in $[a, b]$ and substituting for $X$ in $\int_{a}^{b} d H^{a} \cdot X$, it is seen that $\int_{a}^{b} d H \cdot X=C$. Thus $X$ satisfies $\left(^{*}\right)$ for this $g$ and $C$. Suppose $Y$ is in $C_{[a, b]}$ and satisfies $\left({ }^{*}\right)$. Then, by the above lemma,

$$
Q Y(a)=C-\int_{a}^{b}\left[\int_{j}^{b} d H(s) \cdot M(s, j)\right] d g
$$


and so $Y(a)=U$ which means that $Y(t)=U+g(t)-g(u)+\int_{a}^{t} d F \cdot Y$ and hence by (iii), $X=Y$. Thus if $Q$ takes $S$ onto $S$ reversibly, there is a unique solution to (*) for each $g$ in $C_{[a, b]}$ and $C$ in $S$.

THEOREM B. If $\int_{a}^{b} d H \cdot M(j, a)$ has a bounded inverse which takes $S$ onto $S$, that is, if $\left[\int_{a}^{b} d H \cdot M(j, a)\right]^{-1}$ is in $B$, then there is a. function $R$ from $[a, b]$ to $B$ and a function $K$ from $[a, b] \times[a, b]$ to $B$ such that if $g$ is in $C_{[a, b]}$ and $C$ is in $S$, then the only element $Y$ of $C_{[a, b]}$ satisfying $\left({ }^{*}\right)$ for each of $t$ and $u$ in $[a, b]$ is given by $Y(t)=$ $R(t) C+\int_{a}^{b} K(t, j) d g$ for each $t$ in $[a, b]$. Moreover, such a pair of functions $R$ and $K$ is given by $R(t)=\left[\int_{a}^{b} d H \cdot M(j, t)\right]^{-1}$ and

$$
K(t, u)= \begin{cases}-\left[\int_{a}^{b} d H \cdot M(j, t)\right]^{-1} \int_{u}^{b} d H \cdot M(j, u)+M(t, u) & \text { if } a \leqq u \leqq t \\ -\left[\int_{a}^{b} d H \cdot M(j, t)\right]^{-1} \int_{u}^{b} d H \cdot M(j, u) & \text { if } t \leqq u \leqq b .\end{cases}
$$

Proof. Suppose that $g$ is in $C_{[a, b]}$ and $C$ is in $S$. From Theorem A, $\left({ }^{*}\right)$ has a unique solution $Y$ for this $C$ and $g$, and from the lemma in the proof of Theorem A,

$$
\left[\int_{a}^{b} d H \cdot M(j, a)\right] X(a)=C-\int_{a}^{b}\left[\int_{j}^{b} d H(s) \cdot M(s, j)\right] d g
$$

and so

$$
X(a)=\left[\int_{a}^{b} d H \cdot M(j, a)\right]^{-1}\left\{C-\int_{a}^{b}\left[\int_{j}^{b} d H(s) \cdot M(s, j)\right] d g\right\} .
$$

Using (iii) and the fact that

$$
\begin{gathered}
M(t, a)\left[\int_{a}^{b} d H \cdot M(j, a)\right]^{-1}=\left[\int_{a}^{b} d H \cdot M(j, t)\right]^{-1} \\
X(t)=\left[\int_{a}^{b} d H \cdot M(j, t)\right]^{-1} C-\int_{a}^{b}\left\{\left[\int_{a}^{b} d H \cdot M(j, t)\right]^{-1} \int_{j}^{b} d H(s) \cdot M(s, j)\right\} d g \\
+\int_{a}^{t} M(t, j) d g \\
=R(t) C+\int_{a}^{b} K(t, j) d g
\end{gathered}
$$

where $R$ and $K$ are defined as in the statement of the theorem.

3. A nonlinear boundary value problem. Here a problem is considered which includes the one in the preceding section. Essentially, the requirements of $\S 2$ that each of $F(t)$ and $H(t)$ be an element of $B$ for every $t$ in $[a, b]$ and that $F$ and $H$ be of bounded variation are 
replaced by considerably weaker conditions. Theorem $D$ gives a necessary and sufficient condition for the nonlinear problem considered to have a unique solution. First a fundamental theorem for a certain type of integral equation is given.

THeORem C. Suppose that $[a, b]$ is a number interval and $F$ is a function from $[a, b]$ to $B^{*}$ such that if $A$ is in $S$ and $r>0$, there is a variation function $U$ on $[a, b]$ and a variation function $V$ on $[a, b]$ such that

$$
\|[F(p)-F(q)] x\| \leqq U(p, q)
$$

and

$$
\|[F(p)-F(q)] x-[F(p)-F(q)] y\| \leqq V(p, q)\|x-y\|
$$

if each of $p$ and $q$ is in $[a, b],\|A-x\| \leqq r$ and $\|A-y\| \leqq r$. Then, if $c$ is in $[a, b]$, there is a segment $Q^{\prime}$ containing $c$ such that if $Q$ is the common part of $Q^{\prime}$ and $[a, b]$, there is only one continuous function $Y$ from $Q$ to $S$ such that $Y(t)=A+\int_{c}^{t} d F \cdot Y$ if $t$ is in $Q$.

This follows from Theorem $\mathrm{F}$ of [3].

Definition. Suppose $F$ is a function from $[a, b]$ to $B^{*}$ and $c$ is in $[a, b]$. If there is a point $A$ in $S$ and an element $Y$ of $C_{[a, b]}$ such that $Y(t)=A+\int_{c}^{t} d F \cdot Y$ for each $t$ in $[a, b]$, then the set which contains only each such point $A$ is denoted by $F_{c:[a, b]}$.

Lemma 4.1. Suppose that $F$ satisfies the hypothesis of Theorem $C$ and for some number $c$ in $[a, b]$ and that there is a segment $Q^{\prime}$ as in the theorem which has $[a, b]$ as subset. Then, for each number $u$ in $[a, b]$, there is a set $F_{u ;[a, b]}$.

Proof. Given such a number $c$ and segment $Q^{\prime}$, then $Q=[a, b]$ and there is a point $A$ in $S$ and an element $Y$ of $C_{[a, b]}$ such that $Y(t)=A+\int_{c}^{t} d F \cdot Y$ for each $t$ in $[a, b]$. Thus if $u$ is in $[a, b], Y(u)=$ $A+\int_{c}^{u} d F \cdot Y$ and $Y(t)=Y(u)+\int_{u}^{t} d F \cdot Y$ for each $t$ in $[a, b]$. Thus there is a set $F_{u:[a, b]}$.

Definition. Suppose the hypothesis of Lemma 4.1 holds. $M$ denotes a function from $[a, b] \times[a, b]$ such that if each of $t$ and $u$ is in $[a, b], M(t, u)$ is the function from $F_{u:[a, b]}$ to $F_{t ;[a, b]}$ such that if $A$ is in $F_{u ;[a, b]}, \quad M(t, u) A$ is $Y(t)$ where $Y$ is the element of $C_{[a, b]}$ satisfying $Y(s)=A+\int_{u}^{s} d F \cdot Y$ for each $s$ in $[a, b]$. 
LEMma 4.2. Under the hypothesis of Lemma 4.1, $M(s, t) M(t, u)=$ $M(s, u)$ for each of $s, t$ and $u$ in $[a, b]$.

Proof. Suppose that each of $s, t$ and $u$ is in $[a, b]$ and $A$ is in $F_{u:[a, b]}$. Then, $Y(s)=A+\int_{u}^{s} d F \cdot Y$ and $Y(t)=A+\int_{u}^{t} d F \cdot Y$ so that $Y(s)=Y(t)+\int_{t}^{s} d F \cdot Y, Y(t)=M(t, u) A$ and $Y(s)=M(s, u) A$. Therefore, $\quad Y(s)=M(t, u) A+\int_{t}^{s} d F \cdot Y$ and $Y(s)=M(s, t)[M(t, u) A]=$ $[M(s, t) M(t, u)] A$. Thus, $M(s, u)=M(s, t) M(t, u)$.

TheOREM D. Suppose that in addition to the hypothesis of Theorem $C$, it is true that for some $c$ in $[a, b]$, there is a set $F_{c ;[a, b]}$. Suppose furthermore that $T$ is a function from $C_{[a, b]}$ to $S$ and that $C$ is in $S$. The following two statements are equivalent:

(i) There is only one element $Y$ of $C_{[p, b]}$ such that

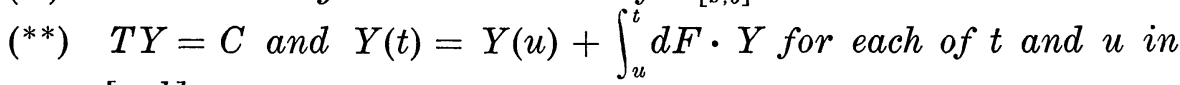
$[a, b]$.

(ii) For some $u$ in $[a, b]$, the function $R$ from $F_{u ;[a, b]}$, defined by $R A=T[M(j, u) A]$ for each $A$ in $F_{u:[a, b]}$ takes only one element of $F_{u ;[a, b]}$ into $C$.

Proof. Suppose that for some $u$ in $[a, b]$, the function $R$ as defined in Theorem $\mathrm{D}$ takes only the point $U$ of $F_{u:[a, b]}$ into $C$. Denote by $Y$ the element of $C_{[a, b]}$ such that $Y(t)=U+\int_{u}^{t} d F \cdot Y$ for each $t$ in $[a, b]$. Thus, $Y(t)=Y(s)+\int_{s}^{t} d F \cdot Y$ and $Y(t)=M^{u}(t, u) U$ for each of $t$ and $s$ in $[a, b]$ and $T Y=T[M(j, u) Y(u)]=C$. Suppose $X$ is in $C_{[a, b]}$ and satisfies $\left({ }^{* *}\right)$. Then, $X(t)=M(t, s) X(s)$ for each of $t$ and $s$ in $[a, b]$ and so $T X=T[M(j, u) X(u)]$ which means that $R[X(u)]=C$ which in turn implies that $X(u)=U$ and so $X(t)=U+\int_{u}^{t} d F \cdot X$ for each $t$ in $[a, b]$. By Theorem B, $X=Y$. Thus the existence of such a $u$ in $[a, b]$ and such a function $R$ implies that $\left({ }^{* *}\right)$ has a unique solution.

Suppose that $\left(^{* *}\right)$ has a unique solution $Y$ which is in $C_{[a, b]}$. Denote by $u$ a number in $[a, b]$. Thus $Y(t)=Y(u)+\int_{u}^{t} d F \cdot Y$ and $Y(t)=$ $M(t, u) Y(u)$ for each $t$ in $[a, b]$ and so $T Y=T[M(j, u) Y(u)]$. Denote by $R$ the function from $F_{u ;[a, b]}$ to $S$ so that $R A=T[M(j, u) A]$ for each $A$ in $F_{u ;[a, b]}$. Thus $R[Y(u)]=C$. Suppose that $V \neq Y(u)$ and $R V=C$. Denote by $X$ the element of $C_{[a, b]}$ so that $X(t)=V+\int_{u}^{t} d F \cdot X$ for each $t$ in $[a, b] . \quad X \neq Y$ as $X(u) \neq Y(u)$. But $X(t)=X(s)+\int_{s}^{t} d F \cdot X$ for each of $t$ and $s$ in $[a, b]$ and $T X=[M(j, u) X(u)]=T[M(j, u) V]=$ $R V=C$, a contradiction. Thus there is not such a point $V$ in $F_{u ;[a, b]}$ and so the existence of a unique element of $C_{[a, b]}$ satisfying $\left({ }^{*}\right)$ implies the existence of the required function $R$. 
4. An example. Suppose that $[a, b]$ is a number interval, $S$ the number plane, each of $p$ and $q$ a continuous function from $[a, b]$ to a number set such that $p(t)>0$ for each $t$ in $[a, b]$ and each of $a_{i j}, b_{i j}$ and $c_{i}, i, j=1,2$, a number. The problem of solving

$$
\begin{aligned}
& \left(p y^{\prime}\right)^{\prime} q y=G \\
& a_{11} y(a)+a_{12} p(a) y^{\prime}(a)+b_{11} y(b)+b_{12} p(b) y^{\prime}(b)=c_{1} \\
& a_{12} y(a)+a_{22} p(a) y^{\prime}(a)+b_{21} y(b)+b_{22} p(b) y^{\prime}(b)=c_{2}
\end{aligned}
$$

for each continuous function $G$ from $[a, b]$ to a number set and each ordered number pair $\left(c_{1}, c_{2}\right)$ is equivalent to the problem of finding a function pair $f_{1}, f_{2}$ each of which is from $[a, b]$ to a number set such that

$$
\left[\begin{array}{l}
f_{1}^{\prime} \\
f_{2}^{\prime}
\end{array}\right]=\left[\begin{array}{cc}
0 & 1 / q \\
q & 0
\end{array}\right]\left[\begin{array}{l}
f_{1} \\
f_{2}
\end{array}\right]+\left[\begin{array}{c}
0 \\
G
\end{array}\right]
$$

and

$$
\left[\begin{array}{ll}
a_{11} & a_{12} \\
a_{21} & a_{22}
\end{array}\right]\left[\begin{array}{l}
f_{1}(a) \\
f_{2}(a)
\end{array}\right]+\left[\begin{array}{ll}
b_{11} & b_{12} \\
b_{21} & b_{22}
\end{array}\right]\left[\begin{array}{l}
f_{1}(b) \\
f_{2}(b)
\end{array}\right]=\left[\begin{array}{l}
c_{1} \\
c_{2}
\end{array}\right]
$$

i.e., the problem of finding a continuous function $f$ from $[a, b]$ to $S$ such that

$$
\begin{aligned}
f & =\left[\begin{array}{l}
f_{1} \\
f_{2}
\end{array}\right], \\
f(t) & =f(u)+g(t)-g(u)+\int_{u}^{t} d F \cdot f
\end{aligned}
$$

and

$$
\int_{a}^{b} d H \cdot f=A_{1} f(a)+A_{2} f(b)=C
$$

for each of $u$ and $t$ in $[a, b]$ where $g(t)=\left[\begin{array}{c}0 \\ G(t)\end{array}\right], F(t)$ is the linear transformation from $S$ to $S$ associated with

$$
\left[\begin{array}{ll}
0 & \int_{a}^{t}(1 / p) d j \\
\int_{a}^{t} q d j & 0
\end{array}\right]
$$

for each $t$ in $[a, b]$, each of $A_{1}$ and $A_{2}$ is a linear transformation from $S$ to $S$ with $A_{1}$ associated with $\left[\begin{array}{ll}a_{11} & a_{12} \\ a_{21} & a_{22}\end{array}\right]$ and $A_{2}$ associated with $\left[\begin{array}{ll}b_{11} & b_{12} \\ b_{21} & b_{22}\end{array}\right]$ and $H$ is defined in the following way: $H(a)=N_{b}$, the transformation which takes each point of $S$ into $\left[\begin{array}{l}0 \\ 0\end{array}\right], H(u)=A_{1}$ if $a<u<b$ and $H(b)=A_{1}+A_{2}$. Suppose that $M$ satisfies $M(t, u)=I+\int_{u}^{t} d F \cdot M(j, u)$ for each of $t$ and $u$ in $[a, b]$. From $\S 2$, for $(\delta)$ to have a unique con- 
tinuous solution for each $g$ and each $C$ it is necessary and sufficient that $\int_{a}^{b} d H \cdot M(j, a)=A_{1}+M(b, a) A_{2}$ have an inverse which is from $S$ onto $S_{r}$. Here is $\int_{a}^{b} d H \cdot M(j, a)$ has an inverse, it is from $S$ to $S$ and is bounded

Suppose that $\int_{a}^{b} d H \cdot M(j, a)$ has an inverse, $G$ is a continuous function from $[a, b]$ to a number set, $C$ is in $S$ and $g=\left[\begin{array}{l}0 \\ G\end{array}\right]$. By Theorem $\mathrm{B}$, there is a function $K$ from $[a, b] \times[a, b]$ to $B$ and a function $R$ from $[a, b]$ to $B$ such that $f(t)=R(t) C+\int_{a}^{b} K(t, j) d g$ for each $t$ in $[a, b]$. Denote by each of $R_{i j}, K_{i j}, i, j=1,2$ a function from $[a, b]$ to a number set such that if each of $t$ and $u$ is in $[a, b], R(t)$ is associated with

$$
\left[\begin{array}{ll}
R_{11}(t) & R_{12}(t) \\
R_{21}(t) & R_{22}(t)
\end{array}\right]
$$

and $K(t, u)$ is associated with

$$
\left[\begin{array}{ll}
K_{11}(t, u) & K_{12}(t, u) \\
K_{21}(t, u) & K_{22}(t, u)
\end{array}\right]
$$

Thus, $f_{1}(t)=R_{11}(t) c_{1}+R_{12}(t) c_{2}+\int_{a}^{b} K_{12}(t, j) d G$ for each $t$ in $[a, b]$ and $f_{1}$ " is the unique solution to $(\Delta)$.

\section{REFERENCES}

1. J. S. MacNerney, Stieltjes integrals in linear spaces, Ann. of Math., 61 (1955), 354-367. 2. - Continuous products in linear spaces, J. Elisha Mitchell Sci. Soc., 71 (1955), 185-200.

3. J. W. Neuberger, Continuous products and nonlinear integral equations, Pacific J. Math., 8 (1958), 529-549.

4. H. S. Wall, Concerning continuous continued fractions and certain system of Stieltjes integral equations, Rend. Circ. Mat. Palermo II (2), (1953) 73-84.

5. — Concerning harmonic matrices, Arch. Math., V (1954), 160-167.

The IllinoIs INSTITUTE OF TECHNOLOGY

and The University of TenNessee 


\section{PACIFIC JOURNAL OF MATHEMATICS}

\section{EDITORS}

David GILbarg

Stanford University

Stanford, California

\section{F. H. BRowneLL}

University of Washington

Seattle 5, Washington

\section{A. L. Whiteman}

University of Southern California Los Angeles 7, California

\section{J. PAIGe}

University of California

Los Angeles 24, California

\section{ASSOCIATE EDITORS}
E. F. BECKENBACH
T. M. CHERRY
D. DERRY

\author{
E. HEWITT \\ A. HORN \\ L. NACHBIN
}

\author{
M. OHTSUKA \\ H. L. ROYDEN \\ M. M. SCHIFFER
}

E. SPANIER

E. G. STRAUS

F. WOLF

\section{SUPPORTING INSTITUTIONS}

\author{
UNIVERSITY OF BRITISH COLUMBIA \\ CALIFORNIA INSTITUTE OF TECHNOLOGY \\ UNIVERSITY OF CALIFORNIA \\ MONTANA STATE UNIVERSITY \\ UNIVERSITY OF NEVADA \\ NEW MEXICO STATE UNIVERSITY \\ OREGON STATE COLLEGE \\ UNIVERSITY OF OREGON \\ OSAKA UNIVERSITY \\ UNIVERSITY OF SOUTHERN CALIFORNIA
}

\author{
STANFORD UNIVERSITY \\ UNIVERSITY OF TOKYO \\ UNIVERSITY OF UTAH \\ WASHINGTON STATE COLLEGE \\ UNIVERSITY OF WASHINGTON \\ AMERICAN MATHEMATICAL SOCIETY \\ CALIFORNIA RESEARCH CORPORATION \\ HUGHES AIRCRAFT COMPANY \\ SPACE TECHNOLOGY LABORATORIES \\ NAVAL ORDNANCE TEST STATION
}

\footnotetext{
Mathematical papers intended for publication in the Pacific Journal of Mathematics should be typewritten (double spaced), and the author should keep a complete copy. Manuscripts may be sent to any one of the four editors. All other communications to the editors should be addressed to the managing editor, L. J. Paige at the University of California, Los Angeles 24, California.

50 reprints per author of each article are furnished free of charge; additional copies may be obtained at cost in multiples of 50 .
}

The Pacific Journal of Mathematics is published quarterly, in March, June, September, and December. The price per volume (4 numbers) is $\$ 12.00$; single issues, $\$ 3.50$. Back numbers are available. Special price to individual faculty members of supporting institutions and to individual members of the American Mathematical Society: $\$ 4.00$ per volume; single issues, $\$ 1.25$.

Subscriptions, orders for back numbers, and changes of address should be sent to Pacific Journal of Mathematics, 2120 Oxford Street, Berkeley 4, California.

Printed at Kokusai Bunken Insatsusha (International Academic Printing Co., Ltd.), No. 6, 2-chome, Fujimi-cho, Chiyoda-ku, Tokyo, Japan.

PUBLISHED BY PACIFIC JOURNAL OF MATHEMATICS, A NON-PROFIT CORPORATION

The Supporting Institutions listed above contribute to the cost of publication of this Journal, but they are not owners or publishers and have no responsibility for its content or policies. 


\section{Pacific Journal of Mathematics}

\section{Vol. 10, No. $4 \quad$ December, 1960}

M. Altman, An optimum cubically convergent iterative method of inverting a linear bounded operator in Hilbert space . . . . . . . . . . . . . . . . . . . . . . . . . . 1107

Nesmith Cornett Ankeny, Criterion for rth power residuacity ................. 1115

Julius Rubin Blum and David Lee Hanson, On invariant probability measures I . . . . . 1125

Frank Featherstone Bonsall, Positive operators compact in an auxiliary topology ..... 1131

Billy Joe Boyer, Summability of derived conjugate series . . . . . . . . . . . . . . . . 1139

Delmar L. Boyer, A note on a problem of Fuchs . . . . . . . . . . . . . . . . . 1147

Hans-Joachim Bremermann, The envelopes of holomorphy of tube domains in infinite

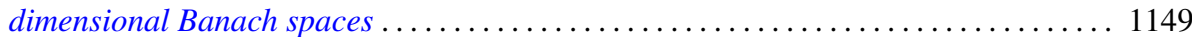

Andrew Michael Bruckner, Minimal superadditive extensions of superadditive

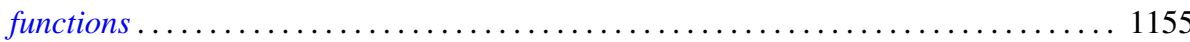

Billy Finney Bryant, On expansive homeomorphisms .................... 1163

Jean W. Butler, On complete and independent sets of operations in finite algebras . . . . . 1169

Lucien Le Cam, An approximation theorem for the Poisson binomial distribution ...... 1181

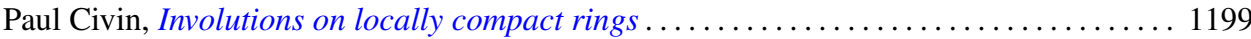

Earl A. Coddington, Normal extensions of formally normal operators . . . . . . . . . 1203

Jacob Feldman, Some classes of equivalent Gaussian processes on an interval ........ 1211

Shaul Foguel, Weak and strong convergence for Markov processes . . . . . . . . . . . 1221

Martin Fox, Some zero sum two-person games with moves in the unit interval ........ 1235

Robert Pertsch Gilbert, Singularities of three-dimensional harmonic functions . . . . . . . 1243

Branko Grünbaum, Partitions of mass-distributions and of convex bodies by

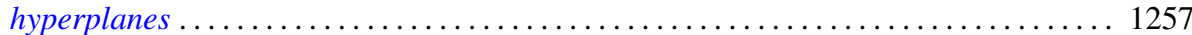

Sidney Morris Harmon, Regular covering surfaces of Riemann surfaces ........... 1263

Edwin Hewitt and Herbert S. Zuckerman, The multiplicative semigroup of integers

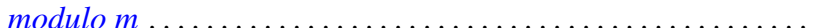

Paul Daniel Hill, Relation of a direct limit group to associated vector groups . ......... 1309

Calvin Virgil Holmes, Commutator groups of monomial groups . .

James Fredrik Jakobsen and W. R. Utz, The non-existence of expansive homeomorphisms

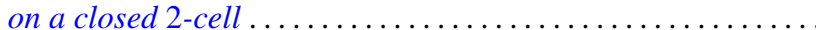

John William Jewett, Multiplication on classes of pseudo-analytic functions . . . . . . . 1323

Helmut Klingen, Analytic automorphisms of bounded symmetric complex domains . . . . 1327

Robert Jacob Koch, Ordered semigroups in partially ordered semigroups . . . . . . . . 1333

Marvin David Marcus and N. A. Khan, On a commutator result of Taussky and

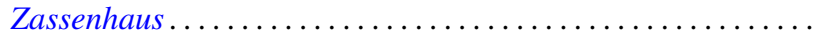

John Glen Marica and Steve Jerome Bryant, Unary algebras......

Edward Peter Merkes and W. T. Scott, On univalence of a continued fraction . . . . . . . 1361

Shu-Teh Chen Moy, Asymptotic properties of derivatives of stationary measures . . . . . 1371

John William Neuberger, Concerning boundary value problems . . . . . . . . . . . 1385

Edward C. Posner, Integral closure of differential rings . . . . . . . . . . . . . . . . . 1393

Marian Reichaw-Reichbach, Some theorems on mappings onto . . . . . . . . . . . . . 1397

Marvin Rosenblum and Harold Widom, Two extremal problems . . . . . . . . . . . . . . . . 1409

Morton Lincoln Slater and Herbert S. Wilf, A class of linear differential-difference

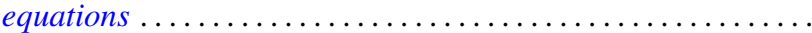

Charles Robson Storey, Jr., The structure of threads . . . . . . . . . . . . . . . . . . 1429

J. François Treves, An estimate for differential polynomials in $\partial / \partial z_{1},, \cdots, \partial / \partial z_{-} n \ldots \ldots 1447$

J. D. Weston, On the representation of operators by convolutions integrals . . . . . . . . 1453

James Victor Whittaker, Normal subgroups of some homeomorphism groups ......... 1469 